Case Report

\title{
Late onset periprosthetic infection of the hip caused by the fish pathogen Lactococcus garvieae in a patient not associated with fish exposure
}

\author{
Marianne Westberg ${ }^{\circledR}$, Hanne Brekke², Nils Olav Hermansen², Bernhard Flatøy ${ }^{1}$ \\ 1. Division of Orthopaedic Surgery, Oslo University Hospital, Oslo, Norway \\ 2. Department of Medical Microbiology, Oslo University Hospital, Oslo, Norway \\ $\triangle$ Corresponding author: Marianne Westberg, MD PhD, Telephone: +47 22118080, E-mail address: marianne.westberg@oslo-universitetssykehus.no \\ (c) The author(s). This is an open access article distributed under the terms of the Creative Commons Attribution License (https://creativecommons.org/licenses/by/4.0/). \\ See http:/ /ivyspring.com/terms for full terms and conditions.
}

Received: 2020.01.05; Accepted: 2020.03.25; Published: 2020.04.06

\begin{abstract}
Lactococcus garvieae is a fish pathogen, rarely causing opportunistic infections in humans. There are only a few cases reported in the literature, mainly endocarditis, suggesting an association with raw fish consumption. We report a case of a periprosthetic hip infection successfully treated with a two-stage revision surgery.
\end{abstract}

Key words: Lactococcus garvieae, fish pathogen, periprosthetic hip infection, two-stage surgery

\section{Introduction}

Lactococcus garvieae (L. garvieae) is traditionally known as a fish pathogen causing septicemia in fish species like: rainbow trout, gray mullet and giant freshwater prawns [1-3]. It can also cause mastitis in ruminants, and has been isolated from dairy products and meat [4-6]. L. garvieae infection in humans have been associated with aquaculture practice $[3,4]$, and with ingestion of contaminated fish [6]. In 1995 Facklam and colleagues suggested that Lactococci were probably opportunistic pathogens mainly affecting persons with underlying debilitations and diseases [7]. In 2016 Yooseok and colleagues reviewed the reported human cases thus far of L. garvieae associated infections, and found that in 19 reported cases nine $(47,4 \%)$ were associated with fish-exposure and 12 $(63,2 \%)$ of the affected patients had a known gastrointestinal disorder [8]. The 1985 Bergey's Manual of Systematic Bacteriology split the Streptococcus genus into three genera: Streptococcus, Enterococcus and Lactococcus. The Lactococcus genus are facultative anaerobic, catalase-negative, and gram-positive cocci, which occur singly, in pairs, or in chains. Several species and subspecies have been described. These bacteria are physiologically similar to the serogroup $\mathrm{D}$ fecal streptococci and resemble enterococci [7]. There have been few reports of human L. garvieae infections in the literature, but in recent years an increasing number of cases have been reported, the majority being endocarditis [9]. There has only been one previous case report of periprosthetic hip infection due to L. garvieae, this was in a fishmonger [10]. We present the first published case of L. garvieae infection in Norway; a periprosthetic late onset hip infection in a patient who denies eating or handling fish.

\section{Case Report}

In 2016, an 80-year old man was admitted to our emergency department due to acute onset of right hip and groin pain for four days. His past medical history included chronic obstructive pulmonary disease (COPD), emphysema grade 3 according to the GOLD classification, as well as hypertension, and hypercholesterolemia. Eight months earlier he was diagnosed with a locally invasive prostate cancer, Gleason score 8, treated with radiotherapy and adjuvant hormone treatment. A right hip 
pertrochanteric fracture had been treated with an osteosynthesis and re-operated due to avascular necrosis 15 and 13 years earlier, respectively. Infection was suspected at the time of revision. The osteosynthesis was hence removed, a resection of the femoral head was performed, and a cement-spacer placed. There was no growth in the perioperatively obtained biopsies, and a cemented total hip arthroplasty (THA) (Spectron ${ }^{\mathrm{TM}}$, Smith \& Nephew) was then performed a few weeks later. The THA had been functioning well over the past 13 years.

On clinical examination he was afebrile, though infection of the THA was suspected. Laboratory tests revealed C-reactive protein (CRP) of $300 \mathrm{mg} / \mathrm{L}$ and leukocyte count of $9.0 \times 10^{9} / \mathrm{L}$. Two sets of blood cultures were negative after seven days of incubation, according to endocarditis guidelines with prolonged incubation. An arthrocentesis of the hip revealed purulent synovial fluid. Microscopy of the synovial fluid showed increased amount of polymorphonuclear leukocytes, supporting the suspicion of an ongoing infectious process. The alfa-defensin test was positive. Radiographic imaging of the right hip revealed signs of loosening of both the stem and the acetabular component, as well as wear of the polyethylene liner. A two-stage exchange arthroplasty was planned. He was operated 6 days after admission with removal of the prosthesis and cement as well as a thorough soft tissue revision. A gentamicin-loaded spacer was placed. Empiric intravenous antimicrobial therapy with vancomycin $1 \mathrm{~g}$ times 2 and cloxacillin 2 $\mathrm{g}$ times 4 per day was started.

The biopsies were cultured aerobically and anaerobically on the following in house produced media: Chocolate blood agar, sheep blood agar, lactose agar, mannitol salt agar, anaerobic blood agar, anaerobic blood agar with kanamycin, and anaerobic blood agar with kanamycin and vancomycin. The media were incubation in $35^{\circ}$ Celcius, $\mathrm{CO} 2$ enriched atmosphere for seven days, according to Norwegian guidelines for culturing biopsies. A semiquantitative measurement method revealed moderate growth of alfa hemolytic grey-white colonies on chocolate and sheep blood agar in six of six biopsy-samples on day one of incubation. On microscopic examination of the growth gram positive short chains of cocci were identified. The microbe was identified as Lactococcus garvieae on matrix-assisted laser desorption/ ionization time of flight mass spectrometry (MALDI-TOF Bruker) with a score of 2,09, with MALDI Biotyper MSP Identification Standard Method 1.1. The identification was further verified by 16s rDNA PCR, where 784 of $784(100 \%)$ base pairs were sequenced without any mismatch, and an NCBI blast identified the microbe as L. garvieae.
Upon identification of the causative microbe, antimicrobial treatment was changed to aminopenicillin according to the pattern of antibiotic susceptibility (Table 1). Antimicrobial susceptibility testing was performed with a quantitative assay for determining the Minimum Inhibitory Concentration (MIC) of antimicrobial agents with Liofilchem MIC Test Strips. Known EUCAST breakpoints were not available for the causative agent. On the fifth post-operative day the patient developed acute on chronic renal failure, with an increase in serum creatinine from $130 \mu \mathrm{mol} / \mathrm{L}$ on admission to 625 $\mu \mathrm{mol} / \mathrm{L}$, a hemodialysis was commenced. It was believed that the development of acute on chronic renal failure was caused by consumption of nephrotoxic medications including vancomycin, NSAIDs and angiotensin 2 blockers, and these medications were stopped. On the $7^{\text {th }}$ postoperative day the haemoglobin level dropped from 9,2 to 5,9 $\mathrm{g} / \mathrm{dL}$, and he received a blood transfusion. Gastroscopy revealed two ulcers, one in the duodenum and one in the antrum. Due to the renal failure the dose of aminopenicillin was reduced to $1 \mathrm{~g}$ times 2 daily.

Table 1. Antimicrobial-susceptibility with determination of MIC for Lactococcus garvieae in the reported case.

\begin{tabular}{ll}
\hline Antibiotic & MIC $(\mathbf{m c g} / \mathbf{m l})$ \\
\hline Ampicillin & 0.25 \\
Ciprofloxacin & 1.0 \\
Clindamycin & $>256$ \\
Ceftriaxon & 0.5 \\
Cefotaxime & 0.5 \\
Gentamicin & 4 \\
Meropenem & 0.064 \\
Penicillin & 0.5 \\
Rifampin & $>32$ \\
Trimetoprim sulpha & 1 \\
Vancomycin & 0.5 \\
\hline MIC was determined with Liofilchen MIC Test strips. (EUCAST breakpoints were \\
not available for L. garvieae.)
\end{tabular}

The patient gradually improved, and was eventually no longer in need of hemodialysis. After 18 days of intravenous antimicrobial treatment, he was transferred to rehabilitation and the treatment was changed to oral aminopenicillin for another 5,5 weeks. The second stage of surgery was performed after a 5-week antibiotic free period. At the time of re-implantation, there were no clinical signs of local infection, and the CRP was normal. He was re-implanted with an uncemented Corail revision stem (Depuy International) and an uncemented trabecular metal cup (ZimmerBiomet). Except for antimicrobial prophylaxis given perioperatively, no additional antimicrobial therapy was given. Six tissue samples were taken during re-implantation, all of which were culture negative after seven days of 
culturing. The recovery was slow and uneventful, with a favourable outcome at 2 years follow-up. There were no clinical signs of infection, radiographicimaging showed no signs of loosening of the prosthesis, and CRP and leukocytes were normal. The patient walked without aid, only restricted by his underlying COPD.

\section{Discussion}

L. garvieae is known to be responsible for septicaemia in various fish species and aquaculture practice. Endemic infections in fish usually occur in the summer, and are suggested to correlate with outbreaks of L. garvieae infections in humans as well. It rarely affects humans, though, and is infrequently found in clinical routine samples. There are only a few reports of L. garvieae related infections in humans in the literature, but the number of reported cases has increased in the last decades. The majority of reported cases are due to bacteremia and endocarditis. Affected patients commonly have multiple comorbidities, especially heart disease and gastrointestinal disorders. To our knowledge there has only been one previous case report of periprosthetic hip infection and one case of knee prosthesis infection due to $L$. garvieae [10,11]. Manipulation and consumption of raw fish have by most authors been thought to be the most likely sources of L. garviaea infections in humans. In both the previous reports of periprosthetic infections, this was the case.

Our patient was an elderly man with a severe COPD and an underlying malignant disease, but with no known heart or gastrointestinal tract diseases prior to admission. He had no recent surgical or dental procedures, or known skin defects. Further he denied any association with handling or consuming raw fish or unpasteurized milk. The periprosthetic hip-infection in this patient occurred during the winter-months. We could not identify the source of infection, neither did the patient have many similarities with previous reported cases of L. garviae infection. He did though develop gastrointestinal bleeding during treatment, which could indicate an underlying occult gastrointestinal ulcer-disease.

Aminopenicillins and cephalosporins are generally effective in the treatment of L. garvieae [12]. A two-stage procedure is regarded as the gold standard in treating periprosthetic infections, and traditionally the success rate of a two-stage procedure is reported to be $80-100 \%$ depending on host factors and bacterial species. The patient responded well to antimicrobial therapy, without signs of relapse at 2-year follow-up.

\section{Conclusion}

Although rarely isolated in human infections, $L$. garvieae may cause severe disease including periprosthetic infections. Despite repeated specific questioning we could not identify the source of the microbe. As the numbers of immunocompromised elderly patients with severe comorbidities are increasing, periprosthetic infections with uncommon low virulence bacteria, like L. garvieae, are more likely to be found, and clinicians and microbiologist should be aware of this. In the presented case, a standard two-stage surgery with a 7-week course of antimicrobial treatment eradicated the infection successfully.

\section{Acknowledgements}

$\mathrm{MW}, \mathrm{HB}$ and $\mathrm{BF}$ initiated the writing of the case report and collected the data. MW wrote the manuscript. $\mathrm{HB}$ and $\mathrm{NOH}$ included the details on microbiological testing. All authors contributed to the final paper and critically reviewed it.

\section{Compliance with ethical standards}

The patient provided a written consent for the use of his clinical data for scientific purposes.

\section{Competing Interests}

The authors have declared that no competing interest exists.

\section{References}

[1] Collins MD, Farrow JAE, Phillips BA, Kandler O. Streptococcus garvieae sp. nov, and Streptococcus plantarum sp.nov. J Gen Microbiol. 1983; 129(11): 3427-3431.

[2] Teixeira LM, Merquior VL, Vianni MC, Carvalho MG, Frcalanza SE, Steigerwalt AG, Brenner DJ, Facklam RR. Phenotypic and genotypic characterization of atypical Lactococcus garvieae strains isolated from water buffalos with subclinical mastitis and confirmation of L.garvieae as a senior subjective synonym of Enterococcus seriolicida. Int J Syst Bacteriol. 1996; 46(3): 664-668.

[3] Vela AI, Vázquez J, Gibello A, Blanco MM, Moreno MA, Liébana P, Albendea C, Alcalá B, Mendez A, Domínquez L, Fernández-Garayzábal JF. Phenotypic and genetic characterization of Lactococcus garvieae isolated in Spain from lactococcus outbreaks and comparison with isolates of other countries and sources. J Clin Microbiol 2000; 38(10): 3791-3795.

[4] Wang CYC, Shie HS, Chen SC, Huang JP, Hsieh IC, Wen MS, Lin FC, Wu D. Lactococcus garviaea infections in humans: possible association with aquaculture outbreaks. Int J Clin Pract 2007; 61(1):68-73.

[5] Yiu KH, Siu CW, To KK, Jim MH, Lee KL, Lau CP, Tse HF. A rare cause of infective endocarditis; Lactococcus garvieae. Int J Cardiol 2007; 114(2): 286-287.

[6] Fortina MG, Ricci G, Foschino R, Picozzi C, Dolci P, Cocolin L, Manachini PL. Phenotypic typing, technological properties, and safety aspects of Lactococcus garviaea strains from dairy environments. J Appl Microbiol 2007; 103:445-453.

[7] Facklam R, Elliot JA. Identification, classification, and clinical relevance of catalase-negative, gram-positive cocci, excluding the streptococci and enterococci. Clin Microbiol Rev 1995; 8: 479-495.

[8] Yooseok S, Min JK, Jae SJ, Yong PC, Chul HK, Youngjin K, Jang WS and Young KY. Afebrile Multi-valve Infective Endocarditis Caused by Lactococcus garvieae: A case Report and Literature Review. Internal Medicine 2016; 55: 1011-1015.

[9] Russo G, Iannetta M, D’Abramo A, Mascellino MT, Pantosti A, Erario L, Tebano G, Oliva A, D’Agostino C, Trinchieri V, Vullo V. Lactococcus garvieae endocarditis in a patient with colonic diverticulosis: first case reporrt in Italy and review of the lilterature. New Microbiol 2012; 35(4): 495-501.

[10] Aubin GG, Bémer P, Guillouzouiz A, Crémet L, Touchais S, Fraquet N, Boutoille D, Reynaud A, Lepelletier D, Corvec S. First report of a hip 
prosthetic joint infection caused by Lactococcus garviaea in a woman fishmonger. J Clin Microbiol 2011; 49: 2074-2076.

[11] Neagoe VI, Zytoun S, Neuhaus HJ. First case report of a late onset knee periprosthetic joint infection caused by Lactococcus garviaea. Case Rep Orthop 2016; 2016:5053640. Epub 2016 Oct 19.

[12] Elliott IA and Facklam RR. Antimicrobial susceptibilities of Lactococcus lactis and Lactococcus garvieae and a proposed method to discriminate between them. J Clin Microbiol 1996; 5: 1296-1297. 